\title{
Oxidative Stress and Dyslipidemia as Indicators of Pathogenesis of Preeclampsia in Pregnant Sudanese Women
}

\author{
Hafiz Ahmed Hobiel' ${ }^{1}$, Hani Yousif Zaki Tadros ${ }^{2}$ \\ ${ }^{1}$ Department of Biochemistry, Faculty of Medicine and Health Sciences, West Kordofan University, Elnihoud, Sudan \\ ${ }^{2}$ Department of Biochemistry and Nutrition, Faculty of Medicine, Gezira University, Medani, Sudan \\ Email: hebiel78@yahoo.com
}

How to cite this paper: Hobiel, H.A. and Tadros, H.Y.Z. (2018) Oxidative Stress and Dyslipidemia as Indicators of Pathogenesis of Preeclampsia in Pregnant Sudanese Women. Journal of Biosciences and Medicines, 6, 48-65.

https://doi.org/10.4236/jbm.2018.67005

Received: May 28, 2018

Accepted: July 24, 2018

Published: July 27, 2018

Copyright $\odot 2018$ by authors and Scientific Research Publishing Inc. This work is licensed under the Creative Commons Attribution International License (CC BY 4.0).

http://creativecommons.org/licenses/by/4.0/

cc) (i) Open Access

\begin{abstract}
Background: Preeclampsia (PE) still ranks as one of obstetrics major problems and is still a serious threat, mainly in underdeveloped countries where its incidence and mortality rates are higher and is a major cause of preterm birth and intra-uterine growth restriction. Aim: To evaluate the role of oxidative stress and dyslipidemia as indicators of pathogenesis and risks of preeclampsia in pregnant Sudanese women attending Wad-Medani Obstetrics and Gynecology Teaching Hospital. Material and Method: This was a cross-sectional (case-control) study carried out in preeclamptic pregnant Sudanese women attending Wad-Medani Obstetrics and Gynecology Teaching Hospital. A total of 208 pregnant women were enrolled in the study, 111 patients and 97 women with normal pregnancy as controls; pregnant women suffering from any systemic or endocrine disorder were excluded. We compared the serum levels of total cholesterol, LDL-cholesterol, HDL-cholesterol, triglycerides, nitric oxide, and total antioxidant capacity between preeclamptic and controls. Result and Conclusion: Pregnant women with increase in BMI have a higher chance of developing PE. Low level of high-density lipoprotein cholesterol and high level of low-density lipoprotein cholesterol define that dyslipidemia increases the risk of PE. Decreased levels of NO and TAC might reflect the oxidative stress and likely contribute to the pathophysiological mechanisms of PE.
\end{abstract}

\section{Keywords}

Preeclampsia, Oxidative Stress, Total Antioxidant Capacity, Nitric Oxide, Dyslipidemia

\section{Introduction}

Pregnancy is one of the most important periods in the life of a woman, a family 
and a society [1]. The success of pregnancy is a result of ongoing interactions between the placenta and the maternal immune and cardiovascular systems [2].

Pregnancy is a transient condition, but when it is complicated by preeclampsia (PE), it has lasting effects on both the mother and the fetus [3].

$\mathrm{PE}$ is a pregnancy multisystem disorder characterized by hypertension ( $\geq 140 / 90 \mathrm{mmHg}$ ), proteinuria ( $\geq 300 \mathrm{mg} / 24$ hrsor $\geq 1+$ dipstick) with or without edema, that appear after 20 weeks of gestation in a previously normotensive non-proteinuric pregnant women [4].

PE found uniquely in the pregnant patient and one that has puzzled scientists for years and one of the "great obstetrical syndromes" [5], is characterized by systemic inflammation, endothelial cell dysfunction, excessive thrombin generation, an anti-angiogenic state and is often associated with multiple organ involvement [6]. However, PE is fundamentally a placental disease which manifests itself, in most cases, by involvement of the vascular (i.e. hypertension) and renal systems (i.e. proteinuria) [7].

During healthy pregnancy, there is an elevation in oxygendemand along with an increase in the production of reactive oxygen species (ROS) to carry out signaling for physiological processes in pregnancy, such as oocyte maturation, ovarian steroidogenesis, ovulation, implantation, blastocyst formation, luteolysis and luteal maintenance [8]. Preeclamptic women can present associated endothelial dysfunction, dyslipidemia and aggravated systemic production of free radicals [9].

$\mathrm{PE}$ is one of the most common complications of human pregnancy with an overall incidence $2 \%-12 \%$ [10] and the syndrome results in more than 63,000 maternal deaths worldwide annually, among which $75 \%$ are experienced in a mild form, and $25 \%$ in a severe form [11]. Prevalence of preeclampsia has increased by up to $30 \%$ over the last decade [12].

The exact cause of $\mathrm{PE}$ remains uncertain. Therefore, PE is still a disease of theories [13]. Although the cause remains largely unknown, the pathogenesis is thought to occur in two main phases. The first stage is asymptomatic, characterized by abnormal placental development during the first trimester resulting in placental insufficiency and the release of excessive amounts of placental materials into the maternal circulation. This in turn leads to the second, symptomatic stage, where in the pregnant woman develops characteristic hypertension, renal impairment, and proteinuria and is at risk for the hemolysis, elevated liver enzymes and low platelet account (HELLP) syndrome, eclampsia, and other end organ damage [14]. At the cellular level, preeclampsia is associated with the release of free radicals by the placenta and therefore generation of oxidative stress. Placenta-borne oxidative and stresses are even sometimes considered as the major molecular determinants of the maternal disease [15].

As PE is considered as a leading cause of maternal mortality and morbidity, measurement of some biochemical markers can enhance the screening and prediction of PE, and therefore the early detection and intervention can limit its progression and harmful effects. 
Research works on the PE and particularly the indicators of pathogenesis are lacking in Sudan. Consequently, this study was conducted to clarify some indicators of pathogenesis of PE in Sudanese preeclamptic women.

Objectives of this study are to estimate the serum lipid profile (TC, TG, LDL, HDL) for evaluating the dyslipidemia in preeclamptic women and to test the association of serum nitric oxide (NO) and total antioxidant capacity (TAC) with the development of preeclampsia.

\section{Material and Methods}

This was a cross-sectional (case-control) study conducted to evaluate the role of oxidative stress and dyslipidemia as indicators of pathogenesis of preeclampsia in pregnant Sudanese women attending Wad-Medani Obstetrics and Gynecology Teaching Hospital from January 2015 to January 2016.

A total of two hundred and eight pregnant women were included in this study. They were selected from the Wards of the Hospital at admission before starting treatment. Informed consent was obtained from each participant before been recruited in to the study. The pregnant women were two groups; patients group of 111 preeclamptic pregnant women, and control group of 97normal pregnant women.

\subsection{Inclusion Criteria}

Preeclamptic women with blood pressure $\geq 140 / 90 \mathrm{mmHg}$, and proteinuria $\geq 300 \mathrm{mg} / 24 \mathrm{hrs}$ or $\geq 1+$ dipstick) were included. The controls were healthy pregnant women with no past history or family history of PE.

\subsection{Exclusion Criteria}

For both patients and controls: pregnant women with pre-gestational cardiac, hepatic or renal disorders, diabetes mellitus, primary or secondary lipid disorders, severe anemia, those who suffer from any other systemic or endocrine disorder were excluded.

A questionnaire was designed to obtain personal data, anthropometric measurements, social and medical information, and clinical investigations.

$5 \mathrm{ml}$ of blood from the median cubital vein was drawn from each participant and putinplain containers, left at room temperature for 30 minutes to clot and then centrifuged for 20 minutes at $5000 \mathrm{rpm}$. Then serum was separated and used for the measurement of TC, LDL, HDL, TG, NO and TAC.

Levels of TC, LDL, HDL, TG were measured using fully automated A15 chemical analyzer manufactured by Biosystems Company S.A Costa Brava 30, 08030 Barcelona (Spain).

The serum NO and total TAC were measured by Huma Reader HS Microtiter plate ELISA Reader manufactured by Bio Assay Systems Company. Corporate Place, Hayward, CA 94545, USA. Website: http://www.biosystems.com/.

Data were statistically analyzed by Statistical Package for the Social Sciences 
(SPSS) software, version 20. The analysis was done by independent student t-test, chi-square test, Pearson's correlation, multiple linear regression. $P$-value $\leq$ 0.05 was considered to be statistically significant.

\section{Results}

The body mass index of preeclamptic women was significantly higher than that of normal pregnant women $(25.33 \pm 0.16$ versus $24.65 \pm 0.18, p=0.005)$ (Figure 1). The systolic blood pressure/diastolic blood pressure of the preeclamptic women showed significant elevation compared to normal pregnant women $(148.83 \pm 0.89 / 96.85 \pm 0.44$ versus $108.04 \pm 0.78 / 74.90 \pm 0.57, p$-values were $<$ 0.001 and $<0.001$ respectively) (Figure 2 ).

Table 1 shows the characteristics of preeclamptic patients and controls regarding residence area and parity. Note: The majority of preeclamptic women have family history of PE (82.9\%), no history of previous $\mathrm{PE}(71.2 \%)$ and late onset of PE (97.3\%).

The biochemical parameters of cases and controls were presented as mean \pm SEM. All the measured parameters were differed significantly between the two study groups (Table 2). The TC, LDL and TG were significantly higher in preeclamptic women than controls ( $p$-values were $0.001,<0.001$ and 0.007 respectively). The HDL level was significantly decreased in preeclamptic patients when compared with control group $(p<0.001)$. Levels of NO and TAC were significantly lower $(p$-values $\leq 0.001)$ in preeclamptic women than control group.

Pregnant women that have high level of TC, LDL, and low level of HDL were

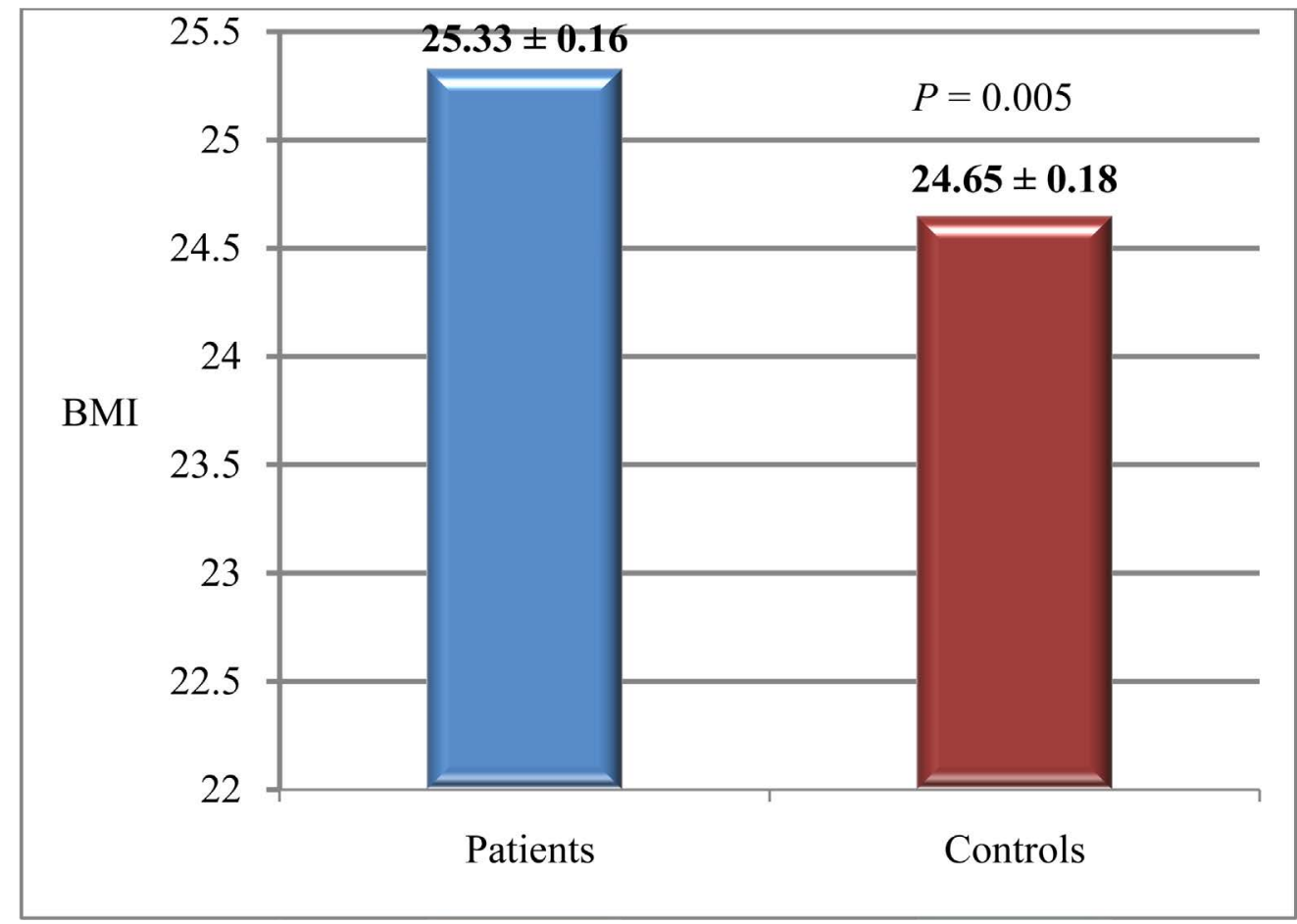

Figure 1. The mean of body mass index of the preeclamptic patients and controls. 


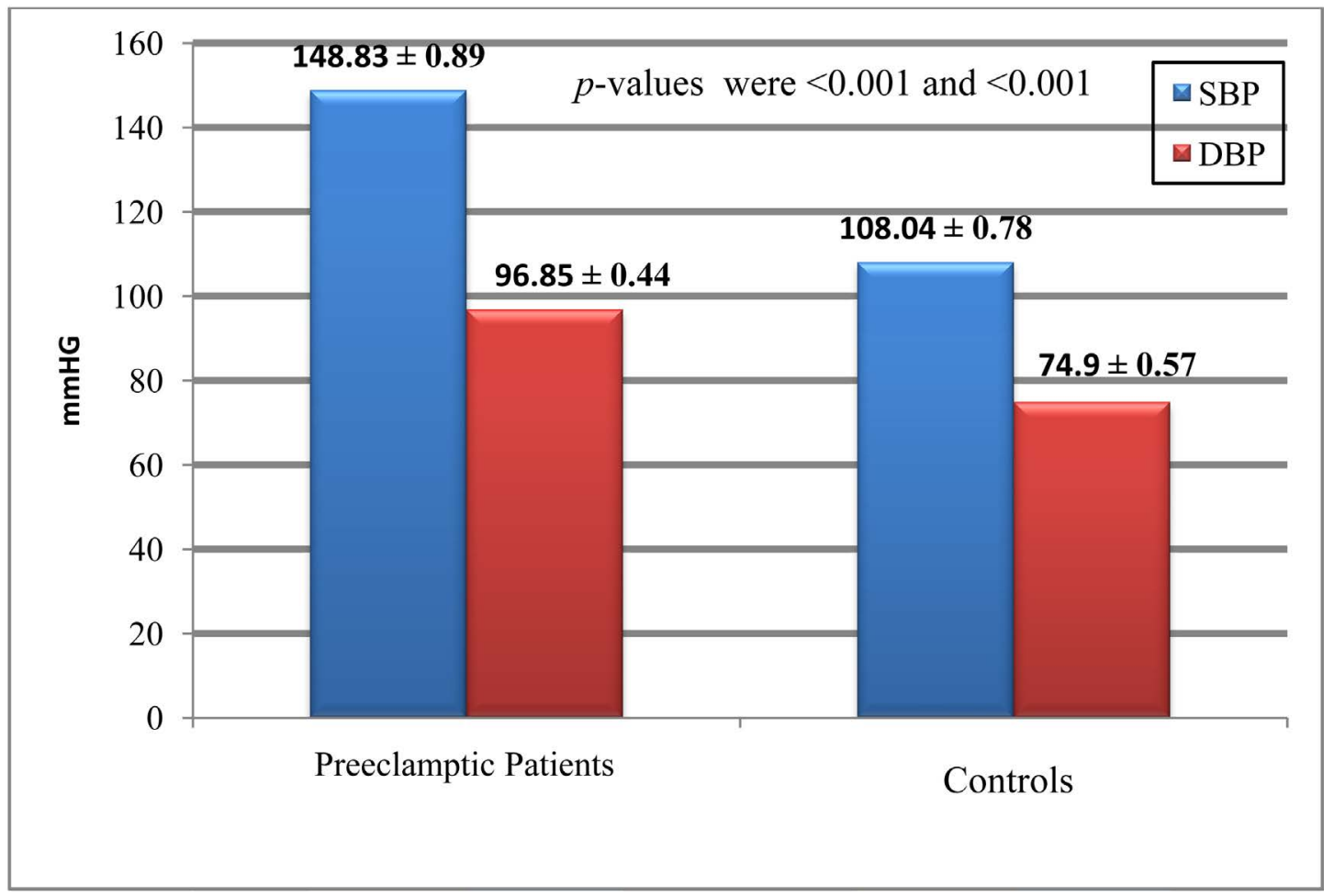

Figure 2. The systolic blood pressure (SBP) and diastolic blood pressure (DBP) of preeclamptic patients and controls.

Table 1. Characteristics and description of study groups.

\begin{tabular}{ccccc}
\hline Characteristic & Patients & Controls & Total & $p$-value \\
\hline Age & & & & \\
& $27.09 \pm 0.62$ & $27.30 \pm 0.62$ & $208(100 \%)$ & 0.811 \\
Gestational age & & & & \\
& $38.16 \pm 0.12$ & $38.76 \pm 0.15$ & $208(100 \%)$ & 0.002 \\
Residence Area & & & & \\
Rural & $64(57.7 \%)$ & $57(58.8 \%)$ & $121(58.2 \%)$ & \\
Urban & $47(42.3 \%)$ & $40(41.2 \%)$ & $87(41.8 \%)$ & 0.889 \\
Total & $111(100 \%)$ & $97(100 \%)$ & $208(100 \%)$ & \\
Parity & & & & \\
Primiparous & $54(48.6 \%)$ & $37(38.1 \%)$ & $91(43.8 \%)$ & \\
Multiparous & $41(36.9 \%)$ & $48(49.5 \%)$ & $89(42.8 \%)$ & \\
Grand multiparous & $16(14.4 \%)$ & $12(12.4 \%)$ & $28(13.5 \%)$ & 0.185 \\
Total & $111(100 \%)$ & $97(100 \%)$ & $208(100 \%)$ & \\
\hline
\end{tabular}

more likely to develop PE compared with pregnant women with normal levels. Preeclamptic women tended to develop oxidative stress by 7.6 times for $\mathrm{NO}$ and 2.6 fold for TAC which in turn leads to development of PE (Table 3).

\subsection{Correlation of Measured Parameters with NO}

Table 4 shows the correlation of BMI, blood pressure, TC, LDL, HDL, TG and 
Table 2. Comparison of biochemical parameters of the study groups.

\begin{tabular}{cccc}
\hline Parameter & Patients $(\mathrm{N}=111)$ & Controls $(\mathrm{N}=97)$ & $p$-value \\
\hline TC & $155.34 \pm 3.64$ & $137.61 \pm 3.68$ & 0.001 \\
LDL & $101.76 \pm 2.43$ & $76.68 \pm 2.29$ & $<0.001$ \\
HDL & $36.91 \pm 0.52$ & $43.22 \pm 0.97$ & $<0.001$ \\
TG & $142.26 \pm 2.68$ & $129.96 \pm 3.66$ & 0.007 \\
NO & $61.69 \pm 1.34$ & $79.33 \pm 1.06$ & $<0.001$ \\
TAC & $230.68 \pm 10.68$ & $291.66 \pm 8.30$ & $<0.001$ \\
\hline
\end{tabular}

Table 3. Comparison of risk estimate considering the parameters of lipid profile, tissue damage and oxidative stress between preeclamptic women and controls.

\begin{tabular}{|c|c|c|c|c|c|c|}
\hline Parameter & Patients & Controls & Total & $p$-value & Chi value & OR (CI: 95\%) \\
\hline \multicolumn{7}{|l|}{$\mathrm{TC}$} \\
\hline Normal & $95(85.6 \%)$ & $87(94.6 \%)$ & $182(89.7 \%)$ & \multirow{4}{*}{0.111} & \multirow{4}{*}{3.243} & \multirow{3}{*}{$2.414(0.904-6.449)$} \\
\hline High & $16(14.4 \%)$ & $5(5.4 \%)$ & $21(10.3 \%)$ & & & \\
\hline Total & $111(100 \%)$ & $92(100 \%)$ & $203(100 \%)$ & & & \\
\hline \multicolumn{5}{|l|}{ LDL } & & \\
\hline Normal & $103(92.8 \%)$ & 95 (99\%) & $198(95.6 \%)$ & \multirow{3}{*}{0.039} & \multirow{3}{*}{4.705} & \multirow{3}{*}{$7.379(0.906-60.103)$} \\
\hline High & $8(7.2 \%)$ & $1(1.0 \%)$ & $9(4.4 \%)$ & & & \\
\hline Total & $111(100 \%)$ & $96(100 \%)$ & $207(100 \%)$ & & & \\
\hline \multicolumn{7}{|l|}{ HDL } \\
\hline Low risk & $73(65.8 \%)$ & $85(89.5 \%)$ & $158(76.5 \%)$ & \multirow{4}{*}{$<0.001$} & \multirow{4}{*}{15.066} & \multirow{4}{*}{$4.070(1.942-8.530)$} \\
\hline High risk & $38(34.2 \%)$ & $10(10.5 \%)$ & $48(23.3 \%)$ & & & \\
\hline Total & $111(100 \%)$ & $95(100 \%)$ & $206(100 \%)$ & & & \\
\hline \multicolumn{4}{|l|}{ TG } & & & \\
\hline Normal & $108(97.3 \%)$ & $84(97.9 \%)$ & $202(97.6 \%)$ & \multirow{3}{*}{0.884} & \multirow{3}{*}{0.062} & \multirow{3}{*}{$1.076(0.607-1.906)$} \\
\hline High & $3(2.7 \%)$ & $2(2.1 \%)$ & $4(2.4 \%)$ & & & \\
\hline Total & $111(100 \%)$ & $96(100 \%)$ & $206(100 \%)$ & & & \\
\hline \multicolumn{7}{|l|}{ NO } \\
\hline Low risk & $33(29.7 \%)$ & $74(76.3 \%)$ & $107(51.4 \%)$ & \multirow{4}{*}{$<0.001$} & \multirow{4}{*}{44.922} & \multirow{4}{*}{$7.605(4.090-14.139)$} \\
\hline High risk & $78(70.3 \%)$ & $23(23.7 \%)$ & $101(48.6 \%)$ & & & \\
\hline Total & $111(100 \%)$ & $97(100 \%)$ & $208(100 \%)$ & & & \\
\hline \multicolumn{4}{|l|}{ TAC } & & & \\
\hline Low risk & $13(11.7 \%)$ & $25(25.8 \%)$ & $38(18.3 \%)$ & \multirow{3}{*}{0.011} & \multirow{3}{*}{6.855} & \multirow{3}{*}{$2.618(1.254-5.464)$} \\
\hline High risk & $98(88.3 \%)$ & $72(74.2 \%)$ & $170(81.7 \%)$ & & & \\
\hline Total & $111(100 \%)$ & $97(100 \%)$ & $208(100 \%)$ & & & \\
\hline
\end{tabular}

Table 4. Correlation of measured parameters with NO.

\begin{tabular}{ccc}
\hline Parameter & Correlation coefficient (r) & $p$-value \\
\hline BMI & -0.075 & 0.285 \\
Systolic blood pressure & $-0.493^{* *}$ & $<0.001$ \\
Diastolic blood pressure & $-0.538^{* *}$ & $<0.001$ \\
\hline
\end{tabular}


Continued

\begin{tabular}{ccc}
\hline TC & $-0.174^{*}$ & 0.013 \\
LDL & $-0.270^{* *}$ & $<0.001$ \\
HDL & $0.180^{* *}$ & 0.009 \\
Triglyceride & -0.133 & 0.055 \\
TAC & $0.181^{* *}$ & 0.009 \\
\hline
\end{tabular}

Correlation is significant at 0.01 level; correlation is significant at 0.05 level, $\mathrm{r}$ : $0.1-0.3$ weak correlation, 0.4 - 0.6 moderate correlation, 0.7 - 0.9 strong correlation.

TAC with NO. NO was negatively correlated with SBP $(\mathrm{r}=-0.493, p \leq 0.001)$, DBP $(\mathrm{r}=-0.538, p \leq 0.00)$, TC $(\mathrm{r}=-0.174, p=0.013)$, LDL $(\mathrm{r}=-0.270, p \leq$ $0.001)$, TG $(\mathrm{r}=-0.133, p=0.055)$, BMI $(\mathrm{r}=-0.075, p=0.285)$, while it was positively correlated with HDL $(\mathrm{r}=0.180, p=0.009)$ and TAC $(\mathrm{r}=0.181, p=0.009)$ Correlation of measured parameters with TAC.

Correlation of BMI, blood pressure, TC, LDL, HDL, LDH and NO with TAC is shown in Table 5. TAC was negatively correlated with SBP $(\mathrm{r}=-0.285, p=$ $<0.001)$, DBP $(\mathrm{r}=-0.268, p \leq 0.001)$, BMI $(\mathrm{r}=-0.063, p=0.365)$, TC $(\mathrm{r}=$ $-0.006, p=0.937)$, LDL $(\mathrm{r}=-0.086, p=0.219)$, while it was positively correlated with HDL $(\mathrm{r}=0.227, p=0.001)$ and $\mathrm{NO}(\mathrm{r}=0.181, p=0.009)$.

\subsection{Multiple Linear Regression Analysis of the PE-Associated Risk and Complication Factors}

Multiple linear regression analysis with forward elimination was conducted to determine among the significant PE-associated risk factors what were the most significant factors that actually contribute to the development of PE as indicated by SBP and DBP.

Because the hypertension is the main clinical manifestation of PE, SBP and DBP were taken as dependent variable with PE-associated risk factors (BMI, lipid profile, TAC and NO) as independent variables of risk estimate. SBP and DBP were taken in two different models.

\subsubsection{Systolic Blood Pressure Model}

From the output of the multiple linear regression for PE-associated risk factors, the most significant factors that predict the development of PE were BMI ( $p=$ $0.036, \beta=0.112)$, LDL $(p \leq 0.001, \beta=0.277), \mathrm{HDL}(p \leq 0.001, \beta$ is -0.328$)$, TAC $(p=0.028, \beta=-0.121)$ and NO $(p \leq 0.001, \beta=-0.318)$ as shown in Table 6 . These results indicate that for an increase of one unit in standard deviations of BMI and LDL the expected increase in standard deviation of SBP was 0.112 and 0.277 respectively. A decrease of one unit in standard deviations of HDL, TAC and NO the expected increase in standard deviation of SBP was $0.328,0.121$ and 0.318 respectively.

The regression lines between SBP and BMI, LDL, HDL, NO, TAC and CK are shown in Figure 3. Although TC and TG were significant PE-associated risk factors, they were eliminated from the multiple linear regression analysis model. 
Table 5. Correlation of measured parameters with TAC.

\begin{tabular}{ccc}
\hline Parameter & Correlation coefficient $(\mathbf{r})$ & $\boldsymbol{p}$-value \\
\hline BMI & -0.063 & 0.365 \\
Systolic blood pressure & $-0.285^{\star *}$ & $<0.001$ \\
Diastolic blood pressure & $-0.268^{\star *}$ & $<0.001$ \\
TC & -0.006 & 0.937 \\
LDL & -0.086 & 0.219 \\
HDL & $0.227^{* *}$ & 0.001 \\
NO & $0.181^{* *}$ & 0.009 \\
\hline
\end{tabular}

Correlation is significant at 0.01 level; correlation is significant at 0.05 level, $\mathrm{r}$ : 0.1 - 0.3 weak correlation, 0.4 - 0.6 moderate correlation, $0.7-0.9$ strong correlation.

Table 6. Multiple linear regression analysis of the biochemical parameters associated with the risks of PE.

\begin{tabular}{ccccccc}
\hline \multirow{2}{*}{$\begin{array}{c}\text { Dependent } \\
\text { variable }\end{array}$} & Parameter & \multicolumn{2}{c}{$\begin{array}{c}\text { Un standardized } \\
\text { coefficient }\end{array}$} & $\begin{array}{c}\text { Standardized } \\
\text { coefficient }\end{array}$ & $\boldsymbol{t}$ & $\boldsymbol{P}$-value \\
\cline { 3 - 5 } & & B & SE & $\boldsymbol{\beta}$ & & \\
\hline \multirow{2}{*}{ SBP } & BMI & 1.405 & 0.666 & 0.112 & 2.111 & 0.036 \\
& LDL & 0.233 & 0.046 & 0.277 & 5.038 & $<0.001$ \\
& HDL & -0.900 & 0.154 & -0.328 & -5.845 & $<0.001$ \\
& TAC & -0.026 & 0.012 & -0.121 & -2.213 & 0.028 \\
& NO & -0.454 & 0.081 & -0.318 & -5.619 & $<0.001$ \\
& BMI & 0.937 & 0.362 & 0.136 & 2.585 & 0.010 \\
& LDL & 0.145 & 0.025 & 0.313 & 5.762 & $<0.001$ \\
DBP & HDL & -0.342 & 0.084 & -0.226 & -4.082 & $<0.001$ \\
& TAC & -0.013 & 0.006 & -0.110 & -2.022 & 0.045 \\
& NO & -0.297 & 0.044 & -0.377 & -6.743 & $<0.001$ \\
\hline
\end{tabular}

B: (slope of regression line) the size of the average difference in the dependent variable that corresponds with a one-unit difference in the independent variable, $\beta$ : the rate of change in dependent variable per unit change in independent variable).

\subsubsection{Diastolic Blood Pressure Model}

From the output of the multiple linear regression for PE-associated risk factors, the most significant predictors for development of PE were BMI $(p=0.010, \beta=$ $0.136)$, LDL $(p \leq 0.001, \beta=0.313)$, HDL $(p \leq 0.001, \beta=-0.226)$, TAC $(p=0.028$, $\beta=-0.110)$ and NO $(p \leq 0.001, \beta=-0.377)$ as presented in Table 6 . These results indicate that for an increase of one unit in standard deviations of BMI and LDL the expected increase in standard deviation of SBP was 0.136 and 0.313 respectively. For a decrease of one unit in standard deviations of HDL, TAC and NO, the expected increase in standard deviation of SBP was $0.226,0.110$ and 0.377 respectively.

The linear relationship between DBP and BMI, LDL, HDL, NO, TAC are shown in Figure 4. 


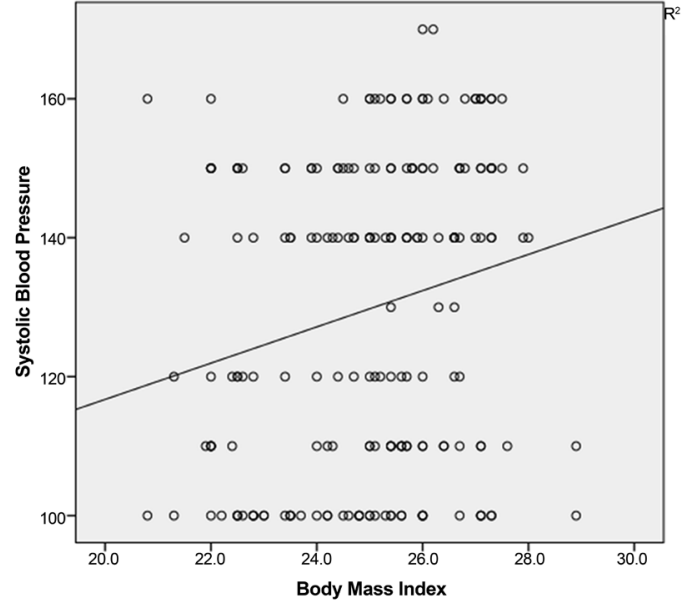

(a)

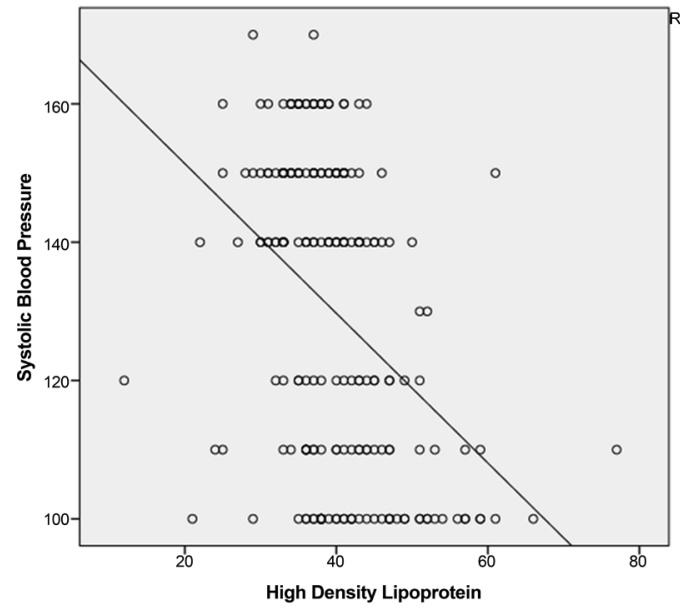

(c)

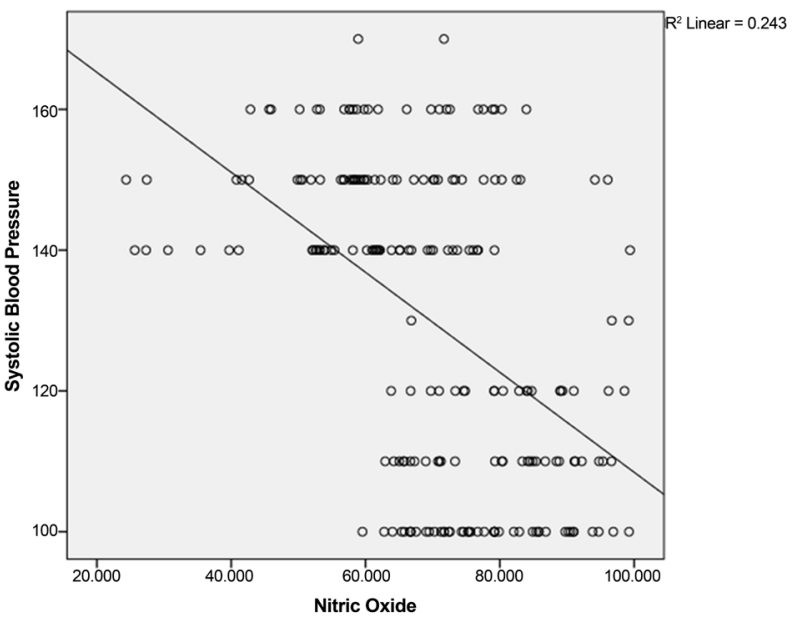

(e)

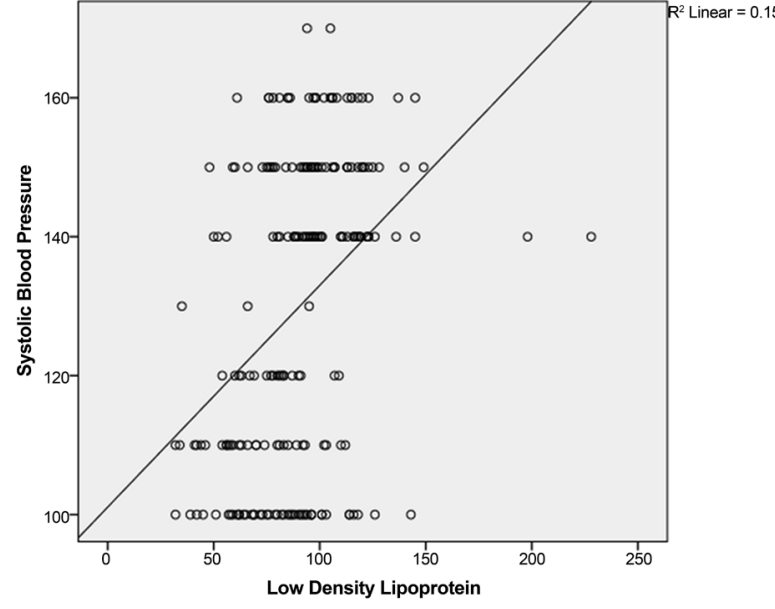

(b)

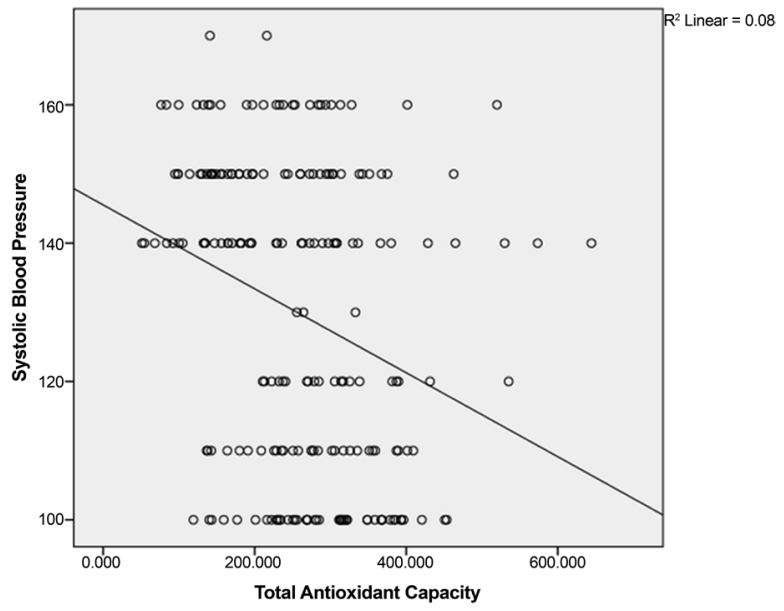

(d)

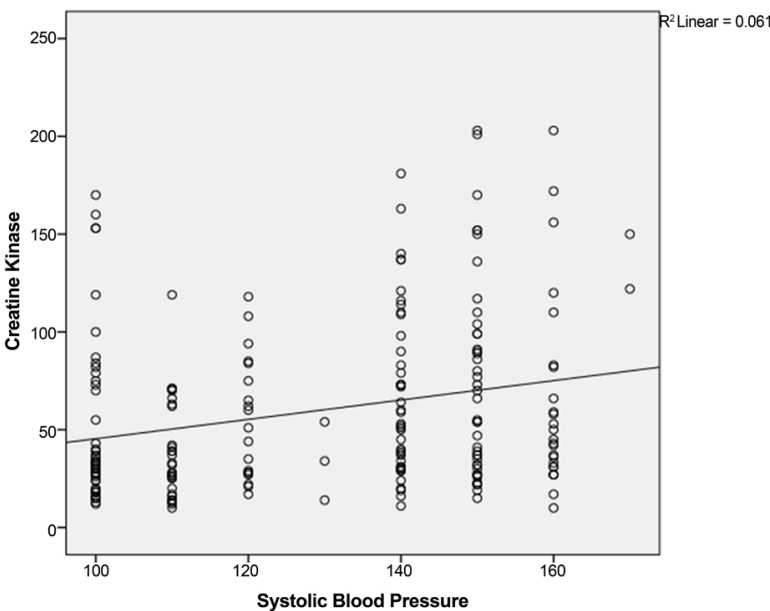

(f)

Figure 3. Linear relationship between SBP and biochemical parameters.

\section{Discussion}

Preeclampsia is a multisystem disorder that remains a major cause of maternal 


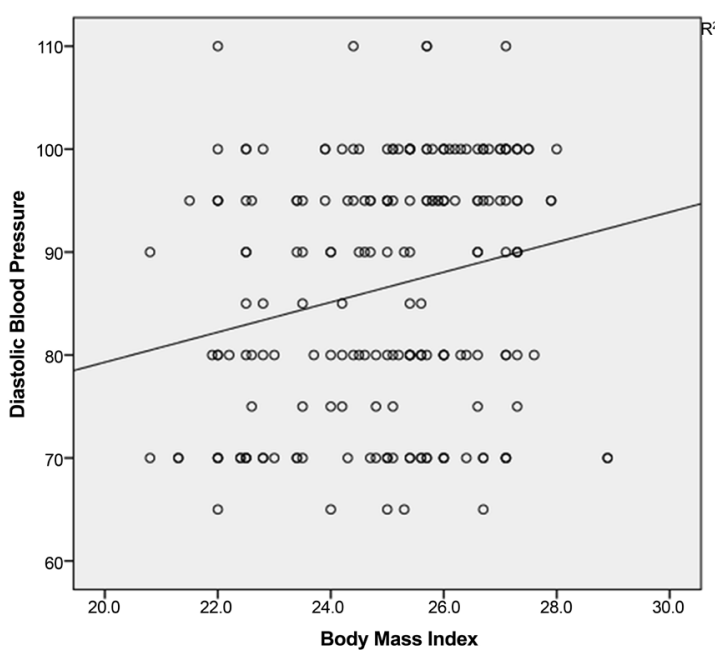

(a)

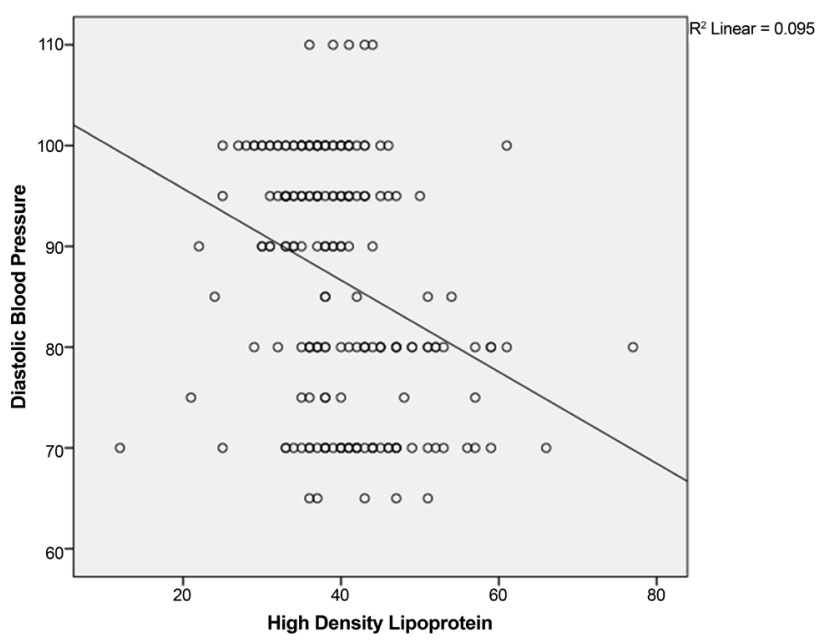

(c)

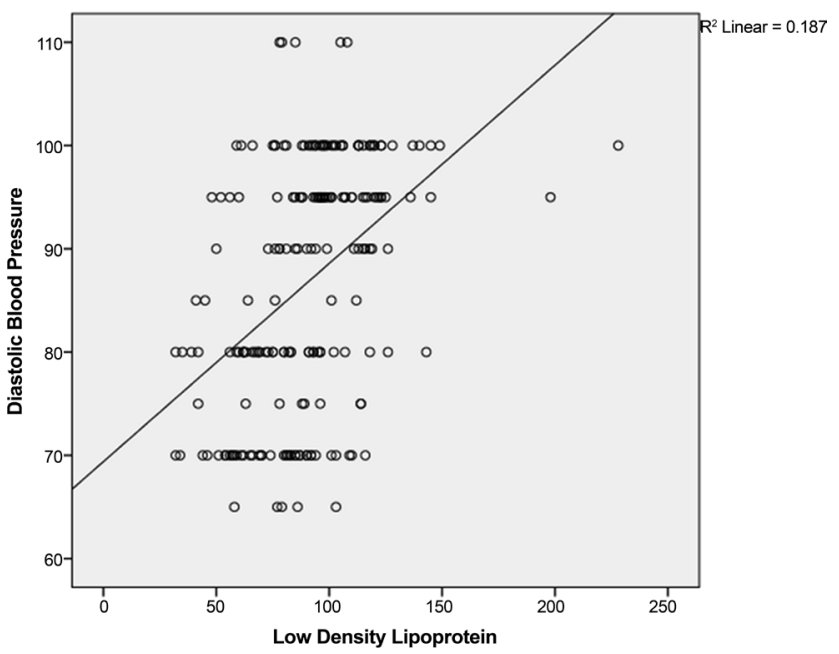

(b)

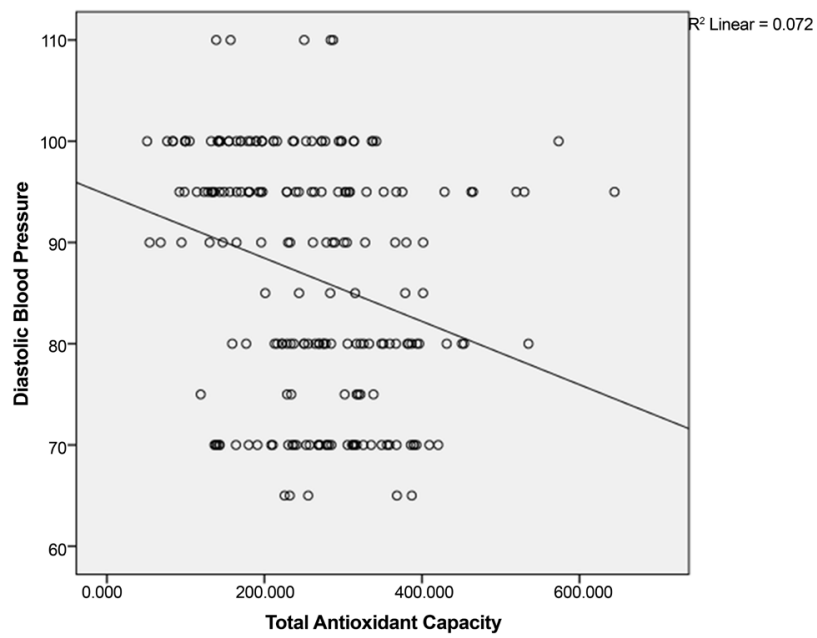

(d)

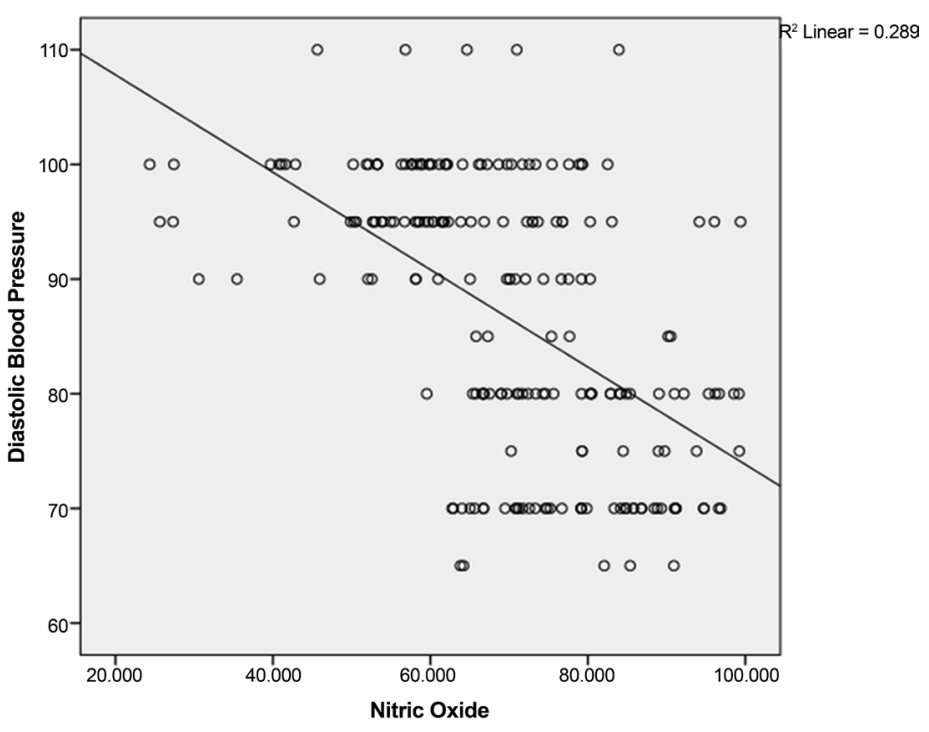

(e)

Figure 4. Linear relationship between DBP and biochemical parameters. 
and fetal morbidity and mortality. Yet, no preventive measures are known to preclude the occurrence of the disease. Free radical damage and several metabolic derangement have been implicated in the pathophysiology of this condition [16]. This study aimed to evaluate the role of oxidative stress, dyslipidemia and markers of tissue damage as indicators of pathogenesis and risk of preeclampsia in pregnant Sudanese women.

In the present study, the ages of the two study groups were not statistically different, while the body mass index was significantly higher in preeclamptic women than control group.

Pregnancy is associated with physiologic hyperlipidemia, and in normal pregnancy this feature is not atherogenic, and attributed to hormonal changes [17]. Pregnancy-related disorders such as PE are associated with a dysregulation of lipid metabolism manifesting in adverse maternal blood lipid levels [18] [19]; in consistency, the present study showed marked increased levels of TC, TG and LDL in preeclamptic women than controls, while preeclamptic women revealed significant decrease in HDL level as compared to controls.

These findings agree with other studies. It was reported that there was a significant increase in TC, LDL and TG with significant decrease in HDL in preeclamptic group compared to normotensive group and stated that dyslipidemia plays an important role in the pathogenesis of PE [20]-[25].

The mechanisms underlying dyslipidemia in PE are implicit. One possibility; inconsequential gestational insulin resistance in PE probably increases the mobilization of fatty acid from visceral adipocytes, leading to over production of VLDL by the liver, and suppresses the activity of lipoprotein lipase, culminating in elevated serum TG concentration and reduced serum HDL level which is major risk factor for vascular dysfunction in PE [26]. Alternatively, PE is a state of hypoestrogenemia, which leads to decreased expression of VLDL/apo E receptors resulting in reduced transport of VLDL to fetal compartment and therefore occurrence of maternal hypertriglyceridemia [3] [27]. Further LDL taken up by the fetus is decreased due to reduced fetoplacental perfusion leading to increased LDL [28]. The elevated TG result in increased atherogenic small dense LDL and reduced HDL levels [29]. For the dyslipidemia and PE association, two mechanismsare suggested: firstly, dyslipidemia may induce endothelial dysfunction secondary to oxidative stress and hence occurrence of PE [30]. Secondly, dyslipidemia may impair trophoblast invasion by the deposition in predisposed vessels, such as the uterine spiral arteries and contributes to the endothelial dysfunction, therefore to a cascade of pathophysiological events of the development of PE [31]. Lipid excess and oxidative stress can provoke endothelial dysfunction. Alterations of the endothelial dysfunction may underlie the hypertension of PE [32]. In Examination of the placental transcriptome, recognized pathways affected by inflammation, lipotoxicity, and oxidative stress were amplified significantly in placenta from obese women. Besides, RNA-seq analysis recognized pronounced decrease in genes related to angiogenesis and hormone activity, in- 
dicating that maternal dyslipidemia can negatively influence mitochondria leading to increased ROS production, oxidative stress and cellular dysfunction [33]. Oxidatively stressed placenta releases a number of trophoblast-derived antiangiogenic (e.g., soluble fms-like tyrosine kinase-1, soluble vascular endothelial growth factor, and soluble endoglin), and proangiogenic (e.g., placenta growth factor) factors that contribute to an exaggerated maternal inflammatory response with generalized endothelial dysfunction [34]. The total cholesterol/high-density lipoprotein ratio (TC/HDL) and the LDL/HDL ratio are two important components and indicators of vascular risk. Interestingly, in this study $32.43 \%$ of preeclamptic patients at atherogenic risk $(\mathrm{T} / \mathrm{HDL})>4.5$; and $35.13 \%$ of patients are defined with high-risk when LDL/HDL $>3.0$ threshold was applied.

$\mathrm{PE}$ is characterized by disturbed extravillous trophoblast migration toward uterine spiral arteries leading to increased uteroplacental vascular resistance and by vascular dysfunction resulting in reduced systemic vasodilatory properties. Its pathogenesis is mediated by an altered bioavailability of $\mathrm{NO}$ and tissue damage caused by increased levels of ROS [35]. Vascular function modulated by the interference of ROS and NO. Increased ROS production seems to suppress the expression and function of endothelial NO synthase [36].

NO exhibits multiple and complex biological functions and many of its effects can be mostly attributed to its strong oxidant capacity. Thus, NO is an important mediator of immune homeostasis and host defense, and changes in its generation or action can contribute to pathologic states [37].

In this study, maternal serum NO levels were significantly low in the preeclamptic women compared to controls. NO was negatively and significantly correlated with SBP, DBP, TC, LDL, TG, LDH, AST, and CK, While, HDL and TAC showed positive and significant correlation. Several reports ascertain the decreased levels of $\mathrm{NO}$ in PE, and its negative correlation with body weight, systolic blood pressure and diastolic blood pressure [38] [39] [40] [41]. In contrast, Norris et al. (1999) reported that the production of NO was significantly higher in the uteroplacental, feto-placental, and peripheral circulation in PE compared to normotensive pregnancies. They attributed the marked increase in NO levels to compensatory mechanism to the pathological effect of PE [42].

In normal pregnancy, NO derived from endothelial nitric oxide synthase (eNOS) contributes to the maintenance of vascular tone to increase uterine blood flow [43]. An up-regulation of eNOS, resulting in increased NO production has been shown to contribute to increases in uteroplacental blood flow via changes in vascular tone [44]. In addition, there is evidence that genetic eNOS polymorphisms may affect the susceptibility to hypertensive disorders of pregnancy [45]. A study on 3 polymorphisms of the eNOS gene and the plasma nitric oxide concentrations was conducted in a population of Chinese origin from mainland China. Two variants 298 Asp allele and eNOS 4a were strongly associated with higher plasma NO concentrations in pregnant women and suggested 
to modulate PE susceptibility [46]. Vascular tone is altered by the interference of ROS and NO, increased ROS production seems to suppress the expression of eNOS and hence reduced production of NO [47]. In fact, several studies have shown that impaired vascular relaxation in $\mathrm{PE}$ has been attributed to reduced bioavailability of NO produced via endothelial NOS [48]. A reduction in the bioavailability of $\mathrm{NO}$ and the imbalance between ROS and antioxidant activity seem to play a critical role in endothelial dysfunction contributing to raised in blood pressure and hence pathogenesis of PE [49] [50]. NO is also a potent inhibitor of platelet aggregation and activation by both cGMP-dependent and -independent mechanisms [51]. Drugs that target various components of the nitric oxide soluble guanylylcyclase pathway can help to increase NO bioavailability, and the delivery of exogenous NO is an attractive therapeutic option [52].

Total antioxidant capacity (TAC) parameter summarizes the overall activity of antioxidants and antioxidant enzymes [53]. Evaluating oxidative stress by measuring TAC can lead to a better understanding of free radical damage in oxidative stress related diseases like PE which would be useful to identify the patients with increased risk of progression of the disease and also for monitoring and optimization of antioxidant therapy [54]. In our study, the serum level of TAC was statistically low in the preeclamptic patients compared to controls. TAC was negatively correlated with SBP, DBP, BMI, TC, LDL and LDH, while it was positively correlated with HDL and NO. Risk estimate considering antioxidants revealed that pregnant women that have low level of NO and TAC had 7.6 and 2.6 times higher risk to develop oxidative stress which in turn leads to development of PE. Study findings are in agreement with the study of Hasan and Dina (2014) who reported that the serum level of TAC was significantly low in preeclamptic cases signifying that the decrease in TAC leads to an imbalance between prooxidants and antioxidants in those women that go on to develop PE [55]. Oxidative stress reflects an imbalance between the formation of oxidative substances and the innate antioxidants that make up the endogenous defense system [56]. During normal pregnancy there is a slight increase in oxidative stress, even in the presence of antioxidant systems [57]. In PE, the abnormal vascular development of the blood vessels in the placenta leads to reduced placental perfusion and induce hypoxia which is by itself a potent stimulus for ROS formation [16].

A genome-wide transcriptomic view identified genes involved in lipid metabolism, angiogenesis, hormone activity, and inflammation to be significantly altered in placenta from obese women. These studies provide evidence for increased lipids and decreased TAC in placenta from obese women, and pinpointed key signaling pathways (increased JNK/FoxO4 signaling) and downstream mediators (HIF-1 $\alpha$ and VEGF-A) that provide a link between maternal-obesity, placental inflammation/oxidative stress, and altered angiogenic factors. Obesity provokes cellular stress, which may in turn adversely affect placental development and function [33]. Antioxidants may be utilized to greater extent to counteract and scavenging free radicals, resulting in the reduction of 
their levels [58].

The limitations of this study include the relatively small sample size. There were no follow up criteria set. Even with this limitation, the findings of this study serve as ground for further studies to better understanding the associations of total antioxidant capacity and nitric oxide levels with preeclampsia.

\section{Conclusions}

Women with excessive BMI change have a higher chance of developing PE. Low level of high-density lipoprotein cholesterol and high level of low-density lipoprotein cholesterol define that dyslipidemia increases the risk of PE. Decreased levels of NO and TAC might reflect the oxidative stress and likely contribute to the pathophysiological mechanisms of PE.

\section{Recommendations}

Longitudinal studies with serial measurements of biochemical markers needed to elucidate the pattern of changes and the pathophysiological consequences of such changes during pregnancy that leads to development of PE. Further studies should be conducted to consider the role of NO and TAC as important factors in the pathogenesis of PE. More studies can clarify the etiological background of PE to provide guidance for the prevention and follow-up of women who experience PE.

\section{References}

[1] Whitehouse, A.J., Robinson, M., Newnham, J.P. and Pennell, C.E. (2012) Do Hypertensive Diseases of Pregnancy Disrupt Neurocognitive Development in Offspring? Paediatric and Perinatal Epidemiology, 26, 101-108.

https://doi.org/10.1111/j.1365-3016.2011.01257.x

[2] James, J.L., Whitley, G.S. and Cartwright, J.E. (2010) Pre-Eclampsia: Fitting Together the Placental, Immune and Cardiovascular Pieces. The Journal of Pathology, 221, 363-378. https://doi.org/10.1002/path.2719

[3] Leeman, L. and Fontaine, P. (2008) Hypertensive Disorders of Pregnancy. American Family Physician, 78, 93-100.

[4] Ghulmiyyah, L. and Sibai, B. (2012) Maternal Mortality from Preeclampsia/Eclampsia. Seminars in Perinatology, 36, 56-59.

https://doi.org/10.1053/j.semperi.2011.09.011

[5] Lin, S., Leonard, D., Co, M.A., Mukhopadhyay, D., Giri, B., Perger, L., Beeram, M.R., Kuehl, T.J. and Uddin, M.N. (2015) Pre-Eclampsia Has an Adverse Impact on Maternal and Fetal Health. Translational Research, 165, 449-463.

https://doi.org/10.1016/j.trsl.2014.10.006

[6] Soto, E., Romero, R., Kusanovic, J.P., Ogge, G., Hussein, Y., Yeo, L., Hassan, S.S., Kim, C.J. and Chaiworapongsa, T. (2012) Late-Onset Preeclampsia Is Associated with an Imbalance of Angiogenic and Anti-Angiogenic Factors in Patients with and without Placental Lesions Consistent with Maternal Underperfusion. The Journal of Maternal-Fetal \& Neonatal Medicine, 25, 498-507. https://doi.org/10.3109/14767058.2011.591461

[7] Ogge, G., Chaiworapongsa, T., Romero, R., Hussein, Y., Kusanovic, J.P., Yeo, L., 
Kim, C.J. and Hassan, S.S. (2011) Placental Lesions Associated with Maternal Underperfusion Are More Frequent in Early-Onset than in Late-Onset Preeclampsia. Journal of Perinatal Medicine, 39, 641-652.

[8] Emet, T., Ustuner, I., Guven, S.G., Balik, G., Ural, U.M., Tekin, Y.B., Senturk, S., Sahin, F.K. and Avsar, A.F. (2013) Plasma Lipids and Lipoproteins during Pregnancy and Related Pregnancy Outcomes. Archives of Gynecology and Obstetrics, 288, 49-55. https://doi.org/10.1007/s00404-013-2750-y

[9] Wild, R., Weedin, E.A. and Wilson, D. (2016) Dyslipidemia in Pregnancy. Endocrinology Metabolism Clinics of North America, 45, 55-63. https://doi.org/10.1016/j.ecl.2015.09.004

[10] Rajaee, M., Nikuei, P., Nejatizadeh, A., Rahimzadeh, M., Massoodi, M., Abedinejad, M., Moradi, S., Mobarkabady, A., Sedigh, B. and Madani, Z. (2015) Prevalence of Preeclampsia in Hormozgan Province. Hormozgan Medical Journal, 18, No. 6.

[11] Duhig, K.E. and Shennan, A.H. (2015) Recent Advances in the Diagnosis and Management of Pre-Eclampsia. F1000Prime Reports, 7, 24.

[12] Leffert, L.R. (2015) What's New in Obstetric Anesthesia? Focus on Preeclampsia. International Journal of Obstetric Anesthesia, 24, 264-271.

[13] Adu-Bonsaffoh, K., Antwi, D.A., Obed, S.A. and Gyan, B. (2015) Nitric Oxide Dysregulation in the Pathogenesis of Preeclampsia among Ghanaian Women. Integrated Blood Pressure Control, 2015, 1-6. https://doi.org/10.2147/IBPC.S68454

[14] Steegers, E.A., von Dadelszen, P., Duvekot, J.J. and Pijnenborg, R. (2010) Pre-Eclampsia. The Lancet, 376, 631-644. https://doi.org/10.1016/S0140-6736(10)60279-6

[15] Aouache, R., Biquard, L., Vaiman, D. and Miralles, F. (2018) Oxidative Stress in Preeclampsia and Placental Diseases. International Journal of Molecular Sciences, 19, 1496. https://doi.org/10.3390/ijms19051496

[16] Rodrigo, R., Parra, M., Bosco, C., Fernandez, V., Barja, P., Guajardo, J. and Messina, R. (2005) Pathophysiological Basis for the Prophylaxis of Preeclampsia through Early Supplementation with Antioxidant Vitamins. Pharmacology \& Therapeutics, 107, 177-197. https://doi.org/10.1016/j.pharmthera.2005.03.001

[17] Winkler, K., Wetzka, B., Hoffmann, M.M., Friedrich, I., Kinner, M., Baumstark, M.W., Zahradnik, H.P., Wieland, H. and Marz, W. (2003) Triglyceride-Rich Lipoproteins Are Associated with Hypertension in Preeclampsia. The Journal of Clinical Endocrinology \& Metabolism, 88, 1162-1166. https://doi.org/10.1210/jc.2002-021160

[18] Xiao, H., Arjun, J., Marc, B., Meike, K., Daniel, S., Peter, B. and Christiane, A. (2013) Increased Placental Phospholipid Levels in Pre-Eclamptic Pregnancies. International Journal of Molecular Sciences, 14, 3487-3499.

[19] Enquobahrie, D.A., Williams, M.A., Butler, C.L., Frederick, I.O., Miller, R.S. and Luthy, D.A. (2004) Maternal Plasma Lipid Concentrations in Early Pregnancy and Risk of Preeclampsia. American Journal of Hypertension, 17, 574-581. https://doi.org/10.1016/j.amjhyper.2004.03.666

[20] Vani, I., Gayathri, A., Nagamani, T. and Sunieeta, H. (2015) Lipid Profile Parameters in Normal and Preeclampsia Complicating Pregnancies-A Prospective Observational Study. The American Journal of Science and Medical Research, 1, 40-43.

[21] Enaruna, N.O., Idemudia, J.O. and Aikoriogie, P.I. (2014) Serum Lipid Profile and Uric Acid Levels in Preeclampsia in University of Benin Teaching Hospital. Nigerian Medical Journal, 55, 423-427.

[22] Singh, U., Yadav, S., Mehrotra, S., Natu, M., Kumari, K. and Yadav, S. (2013) Serum 
Lipid Profile in Early Pregnancy as a Predictor of Preeclampsia. International Journal of Medical Research and Review, 1, 56.

[23] Shalini, M. and Rashmi, R. (2011) Study of Serum Lipid Profile and Magnesium in Normal Pregnancy and Preeclampsia. Asian Journal of Biochemistry, 6, 228-239. https://doi.org/10.3923/ajb.2011.228.239

[24] Pradnya, P. and Mona, T. (2012) Study of Lipid Profile in Preeclampsia. Indian Journal of Basic \& Applied Medical Research, 5, 405-409.

[25] Gohil, J.T., Patel, P.K. and Gupta, P. (2011) Estimation of Lipid Profile in Subjects of Preeclampsia. The Journal of Obstetrics and Gynecology of India, 61, 399-403. https://doi.org/10.1007/s13224-011-0057-0

[26] Gratacos, E., Casals, E., Gomez, O., Llurba, E., Mercader, I., Cararach, V. and Cabero, L. (2003) Increased Susceptibility to Low Density Lipoprotein Oxidation in Women with a History of Pre-Eclampsia. BJOG: An International Journal of Obstetrics and Gynaecology, 110, 400-404. https://doi.org/10.1046/j.1471-0528.2003.02349.x

[27] Aleksandrov, N.A., Fraser, W. and Audibert, F. (2008) Diagnosis, Management, and Evaluation of Hypertensive Disorders of Pregnancy. Journal of Obstetrics and Gynaecology Canada, 30, 1000. https://doi.org/10.1016/S1701-2163(16)32992-9

[28] Seed, P.T., Chappell, L.C., Black, M.A., Poppe, K.K., Hwang, Y.C., Kasabov, N., McCowan, L., Shennan, A.H., Wu, S.H., Poston, L. and North, R.A. (2011) Prediction of Preeclampsia and Delivery of Small for Gestational Age Babies Based on a Combination of Clinical Risk Factors in High-Risk Women. Hypertension in Pregnancy, 30, 58-73. https://doi.org/10.3109/10641955.2010.486460

[29] Romundstad, P.R., Magnussen, E.B., Smith, G.D. and Vatten, L.J. (2010) Hypertension in Pregnancy and Later Cardiovascular Risk: Common Antecedents? Circulation, 122, 579-584. https://doi.org/10.1161/CIRCULATIONAHA.110.943407

[30] Ehrenthal, D.B., Jurkovitz, C., Hoffman, M., Jiang, X. and Weintraub, W.S. (2011) Prepregnancy Body Mass Index as an Independent Risk Factor for Pregnancy-Induced Hypertension. Journal of Women's Health, 20, 67-72. https://doi.org/10.1089/jwh.2010.1970

[31] Chanchal, S., Nagraj, S., Kaushik, G. and Sharma, R. (2015) Study of Serum Lipid Profile AST and LDH in Pre-Elampsia and Eclampsia. International Journal of Advanced Research, 3, 326-329.

[32] Karumanchi, S.A., Maynard, S.E., Stillman, I.E., Epstein, F.H. and Sukhatme, V.P. (2005) Preeclampsia: A Renal Perspective. Kidney International, 67, 2101-2113. https://doi.org/10.1111/j.1523-1755.2005.00316.x

[33] Saben, J., Lindsey, F., Zhong, Y., Thakali, K., Badger, T.M., Andres, A., Gomez-Acevedo, H. and Shankar, K. (2014) Maternal Obesity Is Associated with a Lipotoxic Placental Environment. Placenta, 35, 171-177. https://doi.org/10.1016/j.placenta.2014.01.003

[34] Staff, A.C., Benton, S.J., von Dadelszen, P., Roberts, J.M., Taylor, R.N., Powers, R.W., Charnock-Jones, D.S. and Redman, C.W. (2013) Redefining Preeclampsia Using Placenta-Derived Biomarkers. Hypertension, 61, 932-942. https://doi.org/10.1161/HYPERTENSIONAHA.111.00250

[35] Matsubara, K., Higaki, T., Matsubara, Y. and Nawa, A. (2015) Nitric Oxide and Reactive Oxygen Species in the Pathogenesis of Preeclampsia. International Journal of Molecular Sciences, 16, 4600-4614. https://doi.org/10.3390/ijms16034600

[36] Boger, R.H., Diemert, A., Schwedhelm, E., Luneburg, N., Maas, R. and Hecher, K. (2010) The Role of Nitric Oxide Synthase Inhibition by Asymmetric Dimethylargi- 
nine in the Pathophysiology of Preeclampsia. Gynecologic and Obstetric Investigation, 69, 1-13.

[37] Wander, P. and Jean, S. (2010) The Role of Nitric Oxide in Immune Response Against Trypanosoma Cruzi Infection. The Open Nitric Oxide Journal, 2, 1-6.

[38] Ehsanipoor, R.M., Fortson, W., Fitzmaurice, L.E., Liao, W.X., Wing, D.A., Chen, D.B. and Chan, K. (2013) Nitric Oxide and Carbon Monoxide Production and Metabolism in Preeclampsia. Reproductive Sciences, 20, 542-548. https://doi.org/10.1177/1933719112459231

[39] Sharma, D., Singh, A., Trivedi, S.S. and Bhattacharjee, J. (2011) Intergenotypic Variation of Nitric Oxide and Inflammatory Markers in Preeclampsia: A Pilot Study in a North Indian Population. Human Immunology, 72, 436-439. https://doi.org/10.1016/j.humimm.2011.02.007

[40] Saha, T., Halder, M., Das, A. and Das, S.K. (2013) Role of Nitric Oxide, Angiogenic Growth Factors and Biochemical Analysis in Preeclampsia. Indian Journal of Biochemistry \& Biophysics, 50, 462-466.

[41] Tayal, D., Goswami, B., Patra, S.K., Tripathi, R. and Khaneja, A. (2014) Association of Inflammatory Cytokines, Lipid Peroxidation End Products and Nitric Oxide with the Clinical Severity and Fetal Outcome in Preeclampsia in Indian Women. Indian Journal of Clinical Biochemistry, 29, 139-144. https://doi.org/10.1007/s12291-013-0320-5

[42] Norris, L.A., Higgins, J.R., Darling, M.R., Walshe, J.J. and Bonnar, J. (1999) Nitric Oxide in the Uteroplacental, Fetoplacental, and Peripheral Circulations in Preeclampsia. Obstetrics \& Gynecology, 93, 958-963.

[43] Moncada, S. and Higgs, E.A. (2006) The Discovery of Nitric Oxide and Its Role in Vascular Biology. British Journal of Pharmacology, 147, S193-S201. https://doi.org/10.1038/sj.bjp.0706458

[44] Sandrim, V.C., Palei, A.C., Cavalli, R.C., Araujo, F.M., Ramos, E.S., Duarte, G. and Tanus-Santos, J.E. (2008) eNOS Haplotypes Associated with Gestational Hypertension or Preeclampsia. Pharmacogenomics, 9, 1467-1473. https://doi.org/10.2217/14622416.9.10.1467

[45] Sandrim, V.C., Palei, A.C., Luizon, M.R., Izidoro-Toledo, T.C., Cavalli, R.C. and Tanus-Santos, J.E. (2010) eNOS Haplotypes Affect the Responsiveness to Antihypertensive Therapy in Preeclampsia but Not in Gestational Hypertension. The Pharmacogenomics Journal, 10, 40-45. https://doi.org/10.1038/tpj.2009.38

[46] Chen, Y., Wang, D., Zhou, M., Chen, X. and Chen, J. (2014) Polymorphisms of the Endothelial Nitric Oxide Synthase Gene in Preeclampsia in a Han Chinese Population. Gynecologic and Obstetric Investigation, 77, 150-155.

https://doi.org/10.1159/000358831

[47] Farrow, K., Lakshminrusimha, S., Reda, W., Wedgwood, S., Czech, L., Gugino, S., Davis, J., Russell, J. and Steinhorn, R. (2008) Superoxide Dismutase Restores eNOS Expression and Function in Resistance Pulmonary Arteries from Neonatal Lambs with Persistent Pulmonary Hypertension. American Journal of Physiology-Lung Cellular and Molecular Physiology, 295, L979-L987.

https://doi.org/10.1152/ajplung.90238.2008

[48] Eleuterio, N.M., Palei, A.C., Rangel Machado, J.S., Tanus-Santos, J.E., Cavalli, R.C. and Sandrim, V.C. (2013) Relationship between Adiponectin and Nitrite in Healthy and Preeclampsia Pregnancies. Clinica Chimica Acta, 423, 112-115. https://doi.org/10.1016/j.cca.2013.04.027

[49] Sankaralingam, S., Xu, H. and Davidge, S.T. (2010) Arginase Contributes to Endo- 
thelial Cell Oxidative Stress in Response to Plasma from Women with Preeclampsia. Cardiovascular Research, 85, 194-203. https://doi.org/10.1093/cvr/cvp277

[50] Alpoim, P.N., Godoi, L.C., Freitas, L.G., Gomes, K.B. and Dusse, L.M. (2013) Assessment of L-Arginine Asymmetric 1 Dimethyl (ADMA) in Early-Onset and Late-Onset (Severe) Preeclampsia. Nitric Oxide, 33, 81-82. https://doi.org/10.1016/j.niox.2013.07.006

[51] Crane, M.S., Rossi, A.G. and Megson, I.L. (2005) A Potential Role for Extracellular Nitric Oxide Generation in cGMP-Independent Inhibition of Human Platelet Aggregation: Biochemical and Pharmacological Considerations. British Journal of Pharmacology, 144, 849-859. https://doi.org/10.1038/sj.bjp.0706110

[52] Johal, T., Lees, C.C., Everett, T.R. and Wilkinson, I.B. (2014) The Nitric Oxide Pathway and Possible Therapeutic Options in Pre-Eclampsia. British Journal of Clinical Pharmacology, 78, 244-257. https://doi.org/10.1111/bcp.12301

[53] Prior, R.L., Wu, X. and Schaich, K. (2005) Standardized Methods for the Determination of Antioxidant Capacity and Phenolics in Foods and Dietary Supplements. Journal of Agricultural and Food Chemistry, 53, 4290-4302. https://doi.org/10.1021/jf0502698

[54] Suresh, D. and Annam, V. (2013) Lipid Peroxidation and Total Antioxidant Capacity in Health and Disease-Pathophysiology and Markers: An Overview. International Journal of Medical Science and Public Health, 2, 487-489.

[55] Hasan, F. and Dina, H. (2014) Relationship between Lipid Per Oxidation, Lepton and Lipid Profile in Iraqi Women with Preeclampsia. Engineering and Technology Journals, 32, 225-237.

[56] Buonocore, G., Perrone, S. and Tataranno, M.L. (2010) Oxygen Toxicity: Chemistry and Biology of Reactive Oxygen Species. Seminars in Fetal \& Neonatal Medicine, 15, 186-190. https://doi.org/10.1016/j.siny.2010.04.003

[57] Raijmakers, M., Steegers, E. and Peters, W. (2001) Glutatione S-Transferases and thiol Concentrations in Embryonic and Early Fetal Tissues. Human Reproduction, 16, 2445-2450. https://doi.org/10.1093/humrep/16.11.2445

[58] Manisha, A., Roshan, K. and Sudeep, K. (2015) Study of Lipid Peroxidation and Antioxidant Status in Preeclampsia. Advances in Bioresearch, 6, 144-148. 